\title{
Fbxw7 regulates Notch to control specification of neural precursors for oligodendrocyte fate
}

\author{
Julia L Snyder ${ }^{1,2}$, Christina A Kearns ${ }^{2}$ and Bruce Appel ${ }^{2,3^{*}}$
}

\begin{abstract}
Background: In the developing vertebrate nervous system elevated levels of Notch signaling activity can block neurogenesis and promote formation of glial cells. The mechanisms that limit Notch activity to balance formation of neurons and glia from neural precursors are poorly understood.

Results: By screening for mutations that disrupt oligodendrocyte development in zebrafish we found one allele, called vu56, that produced excess oligodendrocyte progenitor cells (OPCs). Positional cloning revealed that the vu56 allele is a mutation of $f b x w 7$, which encodes the substrate recognition component of a ubiquitin ligase that targets Notch and other proteins for degradation. To investigate the basis of the mutant phenotype we performed in vivo, time-lapse imaging, which revealed that the increase in OPC number resulted from production of extra OPCs by ventral spinal cord precursors and not from changes in OPC proliferation or death. Notch signaling activity was elevated in spinal cord precursors of fbxw7 mutant zebrafish and inhibition of Notch signaling suppressed formation of excess OPCs.

Conclusion: Notch signaling promotes glia cell formation from neural precursors in vertebrate embryos. Our data indicate that Fbxw7 helps attenuate Notch signaling during zebrafish neural development thereby limiting the number of OPCs.
\end{abstract}

Keywords: Notch, Glia, Oligodendrocyte, Myelin, Neural precursor, Zebrafish

\section{Background}

Oligodendrocyte progenitor cells (OPCs), dividing migratory cells that differentiate as myelinating oligodendrocytes, arise from mitotically active neural precursors that occupy distinct regions of the central nervous system (CNS). In the spinal cord, most OPCs arise from the ventrally positioned pMN domain (precursors of motor Neurons), which expresses the Olig1 and Olig2 transcription factors [1-3] and generates motor neurons prior to OPC formation [4-7]. Olig1 and Olig2 are necessary for development of both motor neurons and OPCs $[4,5]$, therefore, regulatory mechanisms must exist to specify Olig $1 / 2^{+}$pMN precursors for either motor neuron or OPC fate. pMN precursors express Ngn1 and Ngn2 transcription factors during neurogenesis and downregulate them prior to gliogenesis [8], suggesting that

\footnotetext{
* Correspondence: bruce.appel@ucdenver.edu

${ }^{2}$ Departments of Pediatrics and Cell and Developmental Biology, University of Colorado School of Medicine, Aurora, CO, USA

${ }^{3}$ University of Colorado School of Medicine, MS 8108, Aurora, CO 80045,

USAFull list of author information is available at the end of the article
}

Ngns inhibit OPC specification. On the other hand, spinal cord precursors initiate expression of transcription factors Sox9, NFIA and NFIB prior to gliogenesis and these factors promote timely formation of oligodendrocytes and astrocytes $[9,10]$, raising the possibility that they contribute to a mechanism that causes neural precursors to switch from neuron to glial cell production.

Signaling mediated by Notch receptors also plays a major role in balancing production of neurons and glia. Generally, loss of Notch signaling in vertebrate embryos results in loss of neural precursors, formation of excess early-born neurons and a deficit of glial cells, including oligodendrocytes [11-17]. This occurs, in part, by elevated expression of the proneural genes $N g n 1$ and $N g n 2$ [18], which promote cell cycle exit and neurogenesis with the consequent loss of later-born glia. Conversely, Notch signaling activity can promote formation of glia in various contexts. In particular, transgene-driven expression of the constitutively active Notch intracellular domain (NICD) in zebrafish during neurogenesis blocked neuron formation and caused a nearly two-fold excess in OPCs, 
which appeared to arise only from pMN precursors and not from other neural precursors [15], indicating that the ability of Notch to promote OPC specification is limited to those precursors that express Olig1/2. Consistent with this, ectopic activation of Notch signaling in the chick spinal cord caused formation of ectopic OPCs only in combination with Olig2 [8]. How Notch activity is controlled to balance neuron and OPC specification remains poorly understood.

By screening for mutations that disrupt oligodendrocyte development we found one allele, vu56, that caused formation of excess OPCs in numbers similar to that produced by NICD expression. Positional cloning revealed that $v u 56$ disrupted $f b x w 7$, which encodes the F-box substrate recognition subunit of an E3 ubiquitin ligase that targets specific proteins, including Notch, for degradation. Our in vivo, time-lapse imaging experiments show directly that the excess OPCs of $f b x w 7^{v u 56}$ mutant zebrafish are produced by pMN precursors. Additionally, our gene expression and functional tests provide evidence that Notch signaling is the principal target of Fbxw7 in pMN precursors for OPC specification. Recent data obtained from mice with brainspecific deletions of $F b x w 7$ indicate that Fbxw7-mediated regulation of Notch and c-Jun is required for neural precursor differentiation and neural cell survival $[19,20]$ and that $F b x w 7$ is required to promote neurogenesis and limit formation of astrocytes [21]. The work presented here now reveals that modulation of Notch signaling activity by Fbxw7 within neural precursors regulates specification of OPC fate.

\section{Results}

\section{Mutation of $f b x w 7$ produces excess oligodendrocyte lineage cells}

By screening for changes in the number and distribution of oligodendrocyte lineage cells marked by $\operatorname{Tg}$ (olig2: EGFP) reporter gene expression, we identified a mutation designated $v u 56$, which, when homozygous, resulted in excess $\mathrm{EGFP}^{+}$dorsal spinal cord cells at 3 days post fertilization (dpf) (Figure 1A, B). Mutant larvae had no apparent morphological defects (Figure 1A', B') but did not inflate swim bladders, which are required for buoyancy and feeding, and died by $9 \mathrm{dpf}$. We verified that $\mathrm{EGFP}^{+}$cells belonged to the oligodendrocyte lineage by performing immunohistochemistry to detect Sox10 protein, which marks both OPCs and myelinating oligodendrocytes in zebrafish [22]. All dorsal spinal cord EGFP ${ }^{+}$cells in vu56 mutant larvae were Sox $10^{+}$, as were ventral $\mathrm{EGFP}^{+}$cells that occupied positions characteristic of OPCs (Figure 1C-D'). Quantification revealed approximately 1.5 fold more Sox $10^{+}$ cells per transverse section taken from vu56 mutant larvae than wild-type larvae. This excess persisted through at least $6 \mathrm{dpf}$ (Figure 1E). To learn if OPCs underwent differentiation we used in situ RNA hybridization to investigate expression of myelin genes. Wild-type larvae expressed plpla and mbp near the pial surface of the spinal cord (Figure 1F, H). vu56 mutant larvae also expressed plp1a and $m b p$ near the pial surface and, occasionally, expressed plp1a at ectopic, medial spinal cord positions (Figure 1G, I). plp $1 a^{+}$and $m b p^{+}$cells were also in excess number in vu56 mutant larvae compared to wild type (Figure 1J and data not shown).

We used simple sequence length polymorphisms (SSLPs) to map the $v u 56$ mutation to a $0.6 \mathrm{cM}$ region of chromosome 1, between the markers z63947 and z10315 (Figure 2A). This region included $f b x w 7$, which we chose as a candidate because expression of human $F B X W 7$ was repressed in glioma [23,24]. Sequencing of PCR products amplified from genomic DNA of vu56 mutant larvae revealed a missense mutation in exon 8 (Figure 2B) predicted to change a neutral glycine residue at amino acid position 261 to a negatively-charged glutamic acid residue near a critical substrate binding site within the second WD repeat (Figure 2A). Both SIFT [25] and PolyPhen [26] programs, which predict the functional effect of amino acid substitutions, identified this mutation as deleterious.

This missense mutation also created a Restriction Fragment Length Polymorphism (RFLP) by changing a BamHI site to a HinfI site. To test linkage between this mutation and the excess oligodendrocyte phenotype, we used the RFLP to genotype larvae produced by intercrosses of heterozygous adults. Whereas phenotypically wild-type larvae were either homozygous for the BamHI allele or heterozygous, all 70 larvae scored as mutant were homozygous for the HinfI allele (Figure 2C and data not shown).

To further validate our identification of $f b w x 7$ as the gene mutated by the vu56 allele, we designed a morpholino oligonucleotide $\left(f b x w 7^{s s M O}\right)$ to bind to the splice acceptor site of exon 4, which contains the F-box domain, with the expectation that it would alter RNA splicing thereby preventing translation of wild-type protein. RNA harvested from $24 \mathrm{hpf}$ embryos and $3 \mathrm{dpf}$ larvae injected with $f b x w 7^{s s M O}$ at one-cell stage produced RT-PCR products with larger molecular weights than control RNA (Figure 2D), suggesting that $f b x w 7^{s s M O}$ prevented excision of intron 3 . We confirmed this by sequencing, which revealed introduction of a premature stop codon before the F-box domain (Figure 2E). We next injected different amounts of $f b x w 7^{\text {sSMO }}$ into newly fertilized $T g$ (olig2:EGFP) embryos. At a 2 ng dose, larvae appeared morphologically normal but had excess dorsal EGFP ${ }^{+}$ spinal cord cells compared to wild type (Figure 2F-G'). Quantification of Sox $10^{+}$cells revealed that the number of oligodendrocyte lineage cells in $f b x w 7^{s s M O}$ injected larvae was similar to that of vu56 mutant larvae (Figure $2 \mathrm{H}-\mathrm{J})$. We conclude that loss of $f b x w 7$ function 


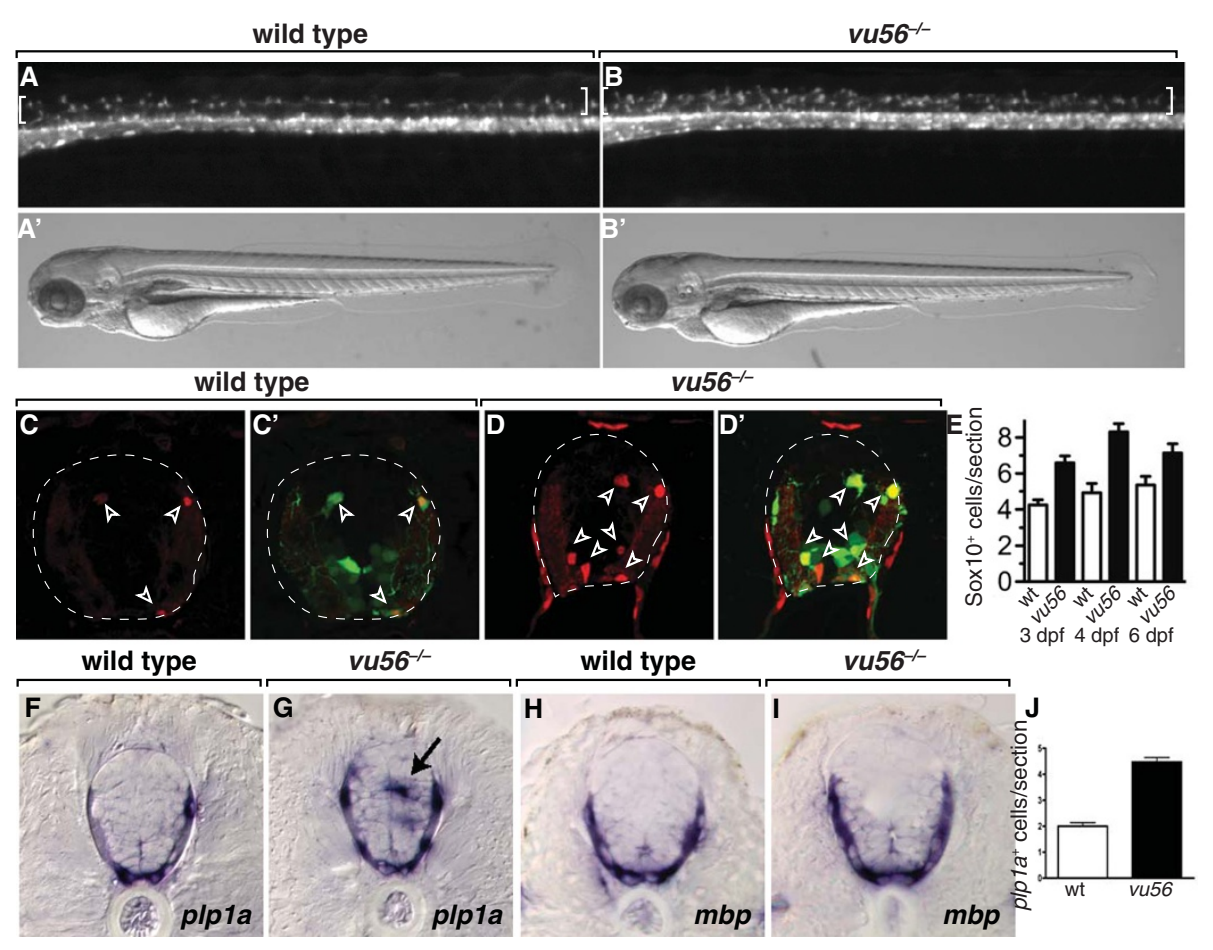

Figure 1 The vu56 mutation causes formation of excess OPCs. (A,B) Lateral images of 3 dpf sibling and vu56 mutants carrying the Tg(olig2: EGFP) reporter marking OPCs in dorsal spinal cord (brackets). Insets show bright field images of living larvae. (C-D') Transverse sections of sibling and vu56 mutant spinal cords processed for immunohistochemistry to detect Sox10 expression (red, arrowheads). Panels $\mathbf{C}^{\prime}$ and $\mathbf{D}^{\prime}$ show Sox 10 labeling merged with olig2:EGFP (green). Dashed lines indicate outer edge of spinal cord. (E) Quantification of Sox $10^{+}$cells per spinal cord section in wild-type (wt) and vu56 mutant larvae at 3, 4, and $6 \mathrm{dpf}(\mathrm{n}=17$ wild-type, 19 mutant at $3 \mathrm{dpf}(p<0.0001), 7$ wild-type and 10 mutant at $4 \mathrm{dpf}$ $(p=0.0002), 5$ wild-type and 5 mutant at $6 \mathrm{dpf}(p=0.0357)$ ). (F-I) Transverse sections of $4 \mathrm{dpf}$ wild-type sibling and vu56 mutant larvae, at the level of trunk spinal cord, processed for in situ RNA hybridization to detect plpla $(\mathbf{F}, \mathbf{G})$ and $m b p(\mathbf{H}, \mathbf{I})$ expression. Arrow indicates ectopic plpla expression. (J) Quantification of plp1a+ oligodendrocytes per section in wild-type and vu56 mutant larvae at $4 \mathrm{dpf}$ ( $\mathrm{n}=4$ larvae per genotype; $p<0.0001)$. Error bars represent SEM.

causes formation of excess oligodendrocytes in zebrafish and hereafter refer to the $v u 56$ allele as $f b x w 7^{\nu u 56}$.

Mammals express three Fbxw7 isoforms, which are produced by splicing of distinct first exons to a common sequence containing 10 exons (Figure 3A) [27]. Isoformspecific exons are transcribed by different promoters, resulting in distinct expression patterns. In adult mice, most tissues express $\alpha$ transcripts, brain expresses $\beta$ transcripts at high level and $\gamma$ transcripts are enriched in heart and skeletal muscle [28]. Encoded within isoformspecific exons are amino acid motifs that determine subcellular localization with $\alpha, \beta$ and $\gamma$ isoforms localizing to nuclei, cytoplasm and nucleoli, respectively [29]. Using a combination of EST clones, PCR to amplify sequences predicted by Ensembl and 5' RACE we identified $\alpha, \beta$ and $\gamma$ transcripts in zebrafish embryos and used these to predict the encoded polypeptides (Figure 3B). The $\alpha$-specific exon encodes a basic domain with similarity to Nuclear Localization Sequences whereas the $\beta$-specific exon, like that of the $\beta$ exon of frogs [30], lacks sequence predicted to encode a hydrophobic transmembrane domain implicated in cytoplasmic localization of the mammalian $\beta$ isoform [29].

We used in situ RNA hybridization to investigate isoform-specific expression in developing zebrafish embryos. Consistent with mammalian expression, somatic mesoderm, which gives rise to muscle, expressed high levels of $\gamma$ transcripts whereas nervous system did not (data not shown). $\alpha$ transcripts were expressed throughout the brain and spinal cord and appeared to be maintained at particularly high levels in medial neural tube, consistent with expression by neural precursors (Figure 2C, E). Neural tissue also expressed $\beta$ transcripts, but by $36 \mathrm{hpf}$ expression appeared to be weak and limited to ventral spinal cord (Figure 3D, F).

\section{Loss of fbxw7 function causes formation of excess OPCs from $\mathrm{pMN}$ precursors}

We identified the source of excess oligodendrocytes in mutant animals using time-lapse imaging. To minimize differences in OPC migration that might result from differences in developmental stage we mounted multiple 

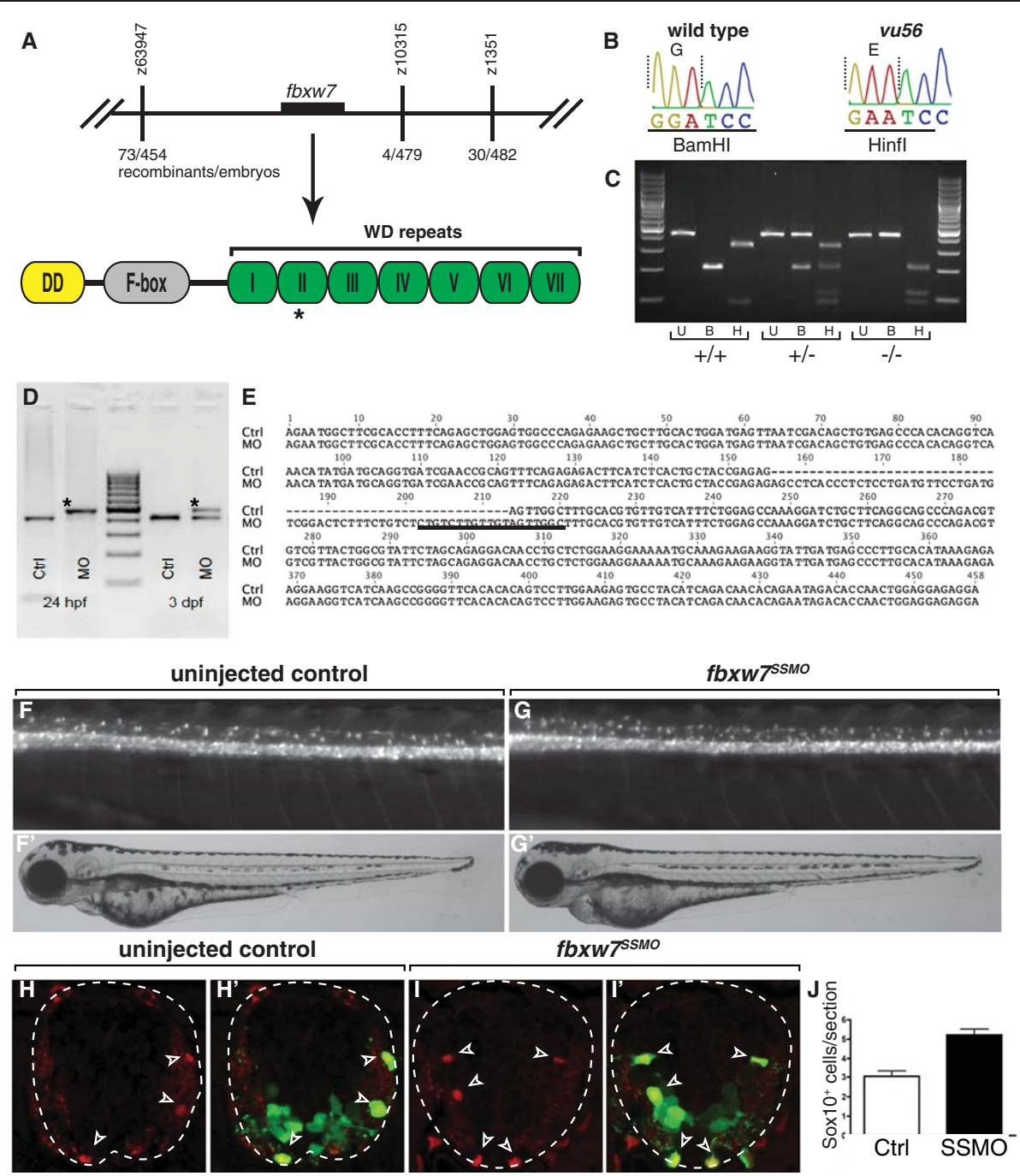

Figure $\mathbf{2} \mathrm{fbxw7}$ is the gene mutated by the vu56 allele. (A) Schematic representation of genetic mapping and sequencing results. The vu56 mutation mapped to a region of Chromosome 1 containing fbxw7. The vu56 mutation created an amino acid substitution within the second WD repeat of the predicted Fbxw7 protein. (B) Sequence traces from wild-type and vu56 mutant cDNAs. The vu56 mutation changed a $\mathrm{G}$ to an A, converting a BamHI restriction site to a Hinfl site. (C) RFLP genotyping of homozygous wild type $(+/+)$, heterozygous (+/-) and homozygous mutant $(-/-)$ larvae. $U=$ uncut, $B=$ BamHI digest, $H=$ Hinfl digest. (D) RT-PCR analysis of fbxw7 mRNA splicing in control (Crtl) and fbxw ${ }^{S S M O}$-injected (MO) $24 \mathrm{hpf}$ embryos and $3 \mathrm{dpf}$ larvae. Upper band in MO lanes (asterisks) indicates splice blocking products. (E) Sequence analysis of RT-PCR products from control embryos, which lack intron 4 sequence (dashed lines) and fbXW $7^{S S M O}$-injected embryos, which retain intron 4 sequence. Underlined sequence is complementary to the splice-blocking morpholino. $(\mathbf{F}, \mathbf{G})$ Lateral images of $3 \mathrm{dpf}$ uninjected control and $f b x W 7^{S S M O}$-injected larvae. Insets show corresponding bright field images. (H-I') Transverse sections of $3 \mathrm{dpf}$ uninjected control and fbxw $7^{S S M O}$ morpholino injected larvae showing Sox10 expression (red, arrowheads). Panels $\mathbf{H}^{\prime}$ and $\mathbf{I}^{\prime}$ show Sox10 labeling merged with olig2:EGFP (green). (J) Quantification of Sox $10^{+}$cells per section in control and fbxw $7^{S S M O}$-injected larvae $(n=8$ larvae each genotype; $p=0.0001)$. Error bars represent SEM.

embryos produced by matings of $f b x w 7^{v u 56+/-} ; \operatorname{Tg}($ olig2: $E G F P$ ) adults in the same imaging chamber, sequentially collected time-lapse image sequences from individual embryos and genotyped them at the end of the imaging period. Between 48 and $63 \mathrm{hpf}$, the period of most ventral to dorsal spinal cord migration, an average of 17 OPCs occupied dorsal spinal cord between somites 5-9 in $f b x w 7^{+/+}$embryos (Figure 4A-A", D). During the same period, an average of 23 and 40 OPCs occupied dorsal spinal cord in $f b x w 7^{+/-}$and $f b x w 7^{-1-}$ embryos, respectively (Figure 4B-C", D). To learn if a change in OPC proliferation also contributed to the difference in OPC number, we counted divisions of dorsally migrating OPCs. Although we found a trend toward slightly more divisions in heterozygous and homozygous mutant larvae than in wild type, the differences did not reach statistical 


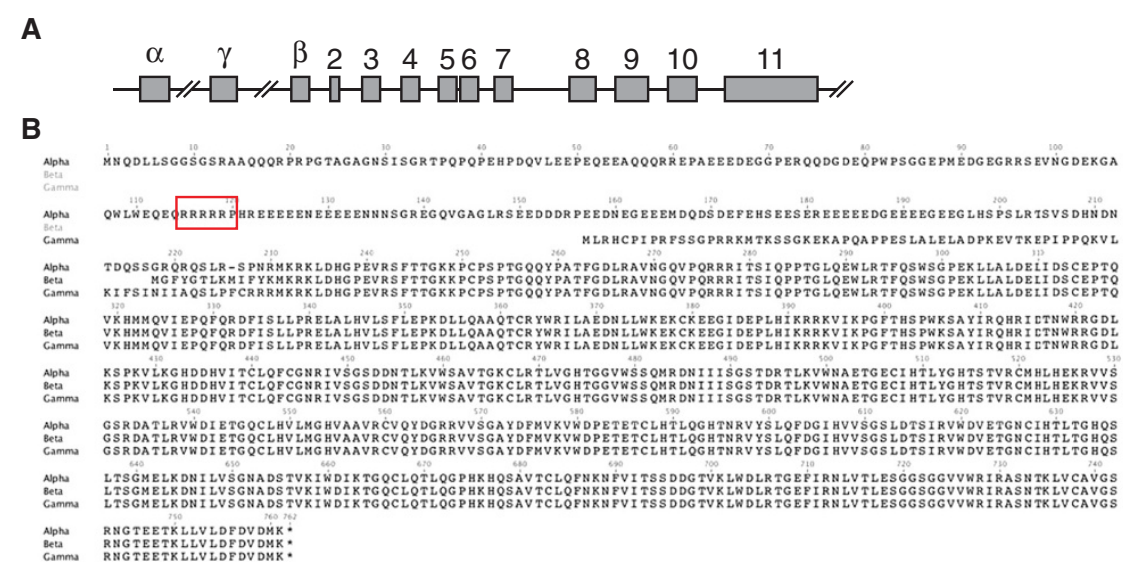

$24 \mathrm{hpf}$ 36 hpf
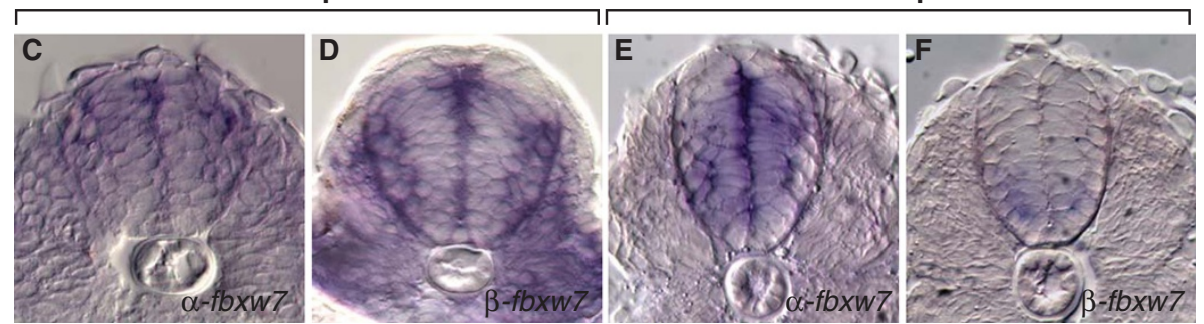

Figure $3 \mathrm{fbxw7}$ gene structure and expression. (A) Schematic representation of fbxw7 gene structure. Three alternative first exons, $a, \gamma$, and $\beta$, are spliced to exons 2-11. (B) Predicted amino acid sequences of Fbxw7 isoforms. The putative nuclear localization sequence of the $a$ isoform is boxed. (C-F) Transverse sections through the level of the trunk spinal cord showing a and $\beta$-specific RNA expression detected by in situ hybridization. At $36 \mathrm{hpf}$, when pMN precursors initiate OPC formation, medial spinal cord cells a-fbxw7 at relatively high level whereas $\beta$-fbxw7 transcripts are only evident at low level in ventral spinal cord.

significance (Figure 4E). Our movies revealed no evidence of cells eliminated by apoptosis, which can be identified by cell fragmentation [31], indicating that differences in cell death do not account for differences in oligodendrocyte number. Therefore, we conclude that the excess oligodendrocytes of $f b x w 7^{v u 56}$ mutant larvae result mostly from excess production of OPCs from ventral spinal cord precursors.

Ventral spinal cord precursors also produce motor neurons, consequently, one possible mechanism that could account for specification of excess OPCs is conversion of cells from motor neuron to OPC fate. To test this we counted motor neurons using immunohistochemistry. At $3 \mathrm{dpf}$, there was no apparent difference in the number of $\mathrm{Isl}^{+}$motor neurons between $f b x w 7^{\text {vu }} 56$ mutant larvae and their wild-type siblings, which would include $f b x w 7^{+/+}$and $f b x w 7^{+/-}$embryos (Figure 4F-H).

An alternative explanation for the formation of excess OPCs is that $f b x w 7^{v u 56}$ mutant larvae have more spinal cord precursors at the time of gliogenesis. To test this we investigated expression of Sox2, a commonly used marker of neural precursors and neural stem cells [32]. By $3 \mathrm{dpf}$, Sox 2 expression in wild-type larvae was mostly limited to cells that lined the central canal, located in ventral spinal cord, including olig2: $\mathrm{EGFP}^{+} \mathrm{pMN}$ precursors (Figure 4I). By contrast, Sox2 was expressed at high level in cells along the entire medial septum and central canal of $3 \mathrm{dpf} f b x w 7^{\text {vu56}}$ mutant larvae (Figure 4J). Both the total number of Sox $2^{+}$cells and the number of Sox $2^{+}$ olig2: $\mathrm{EGFP}^{+}$cells were increased about 1.7 -fold in mutant larvae relative to wild-type larvae (Figure $4 \mathrm{~K}, \mathrm{~L}$ ). As an additional test of neural precursor number we labeled S-phase cells with a brief pulse of BrdU. 3 dpf $f b x w 7^{\text {vu }} 56$ mutant larvae had more than twice the number of $\mathrm{BrdU}^{+}$ and $\mathrm{BrdU}^{+}$olig2:EGFP ${ }^{+}$spinal cord cells than their wild-type siblings (Figure 4M-P). Therefore, $f b x w 7^{v u 56}$ mutant larvae maintained excess number of dividing neural precursors, a subset of which produced excess OPCs.

\section{Fbxw7 limits OPC specification by limiting Notch signaling activity}

Expression of constitutively active Notch1a prior to OPC specification produced an increase in the number of oligodendrocytes nearly identical to that of the $f b x w 7^{v u 56}$ mutation [15]. The signaling active intracellular domain of Notch1 is targeted for degradation by Fbxw7-mediated ubiquitination [33-35]. Therefore, the excess OPCs in 


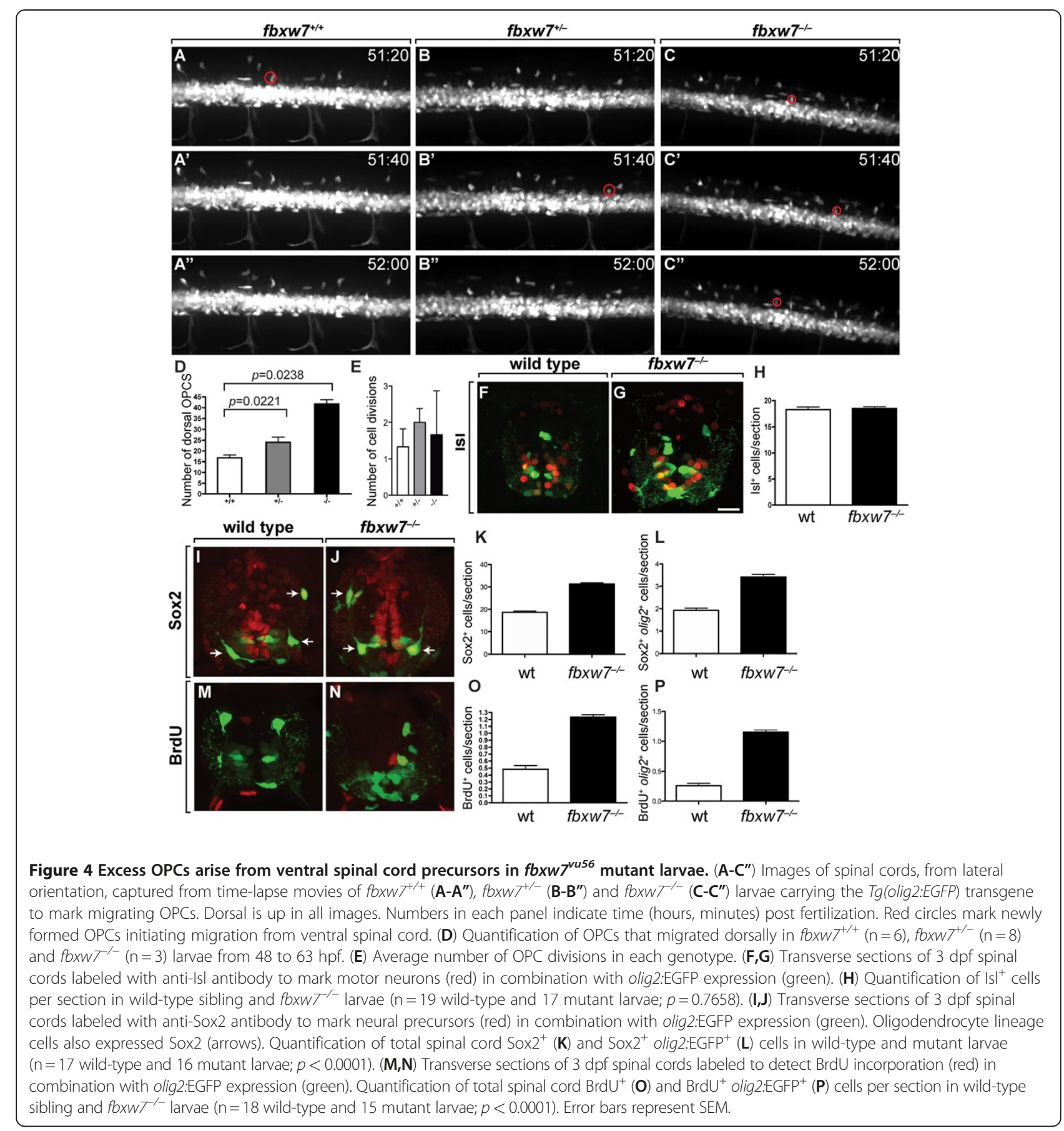

$f b x w 7^{v u 56}$ mutant larvae could result from elevated Notch signaling. As a test of this possibility, we investigated expression of the Notch reporter transgene $\mathrm{Tg}$ (Tp1bglob:hmgb1-mCherry), which drives mCherry expression (hereafter Tp1:mCherry) using a regulatory element containing RBP-JK binding sites [36]. At $3 \mathrm{dpf}$, Tp1:mCherry expression was evident in a few cells near the central canal and medial septum of wild-type larvae and only apparent at low level in olig2:EGFP ${ }^{+}$cells located away from the spinal cord midline (Figure 5A, $\left.\mathrm{A}^{\prime}\right)$. By contrast, numerous cells lining the central canal and medial septum of $f b x w 7^{\text {vu56 }}$ mutant larvae expressed Tp1:mCherry at high level (Figure 5B, B'). Additionally, weaker Tp1:mCherry signal was evident in numerous cells away from the midline, including olig2:EGFP ${ }^{+}$oligodendrocyte lineage cells. At $6 \mathrm{dpf}$, confocal microscope images of wild-type larvae viewed from lateral orientation revealed mCherry expression in scattered cells of 


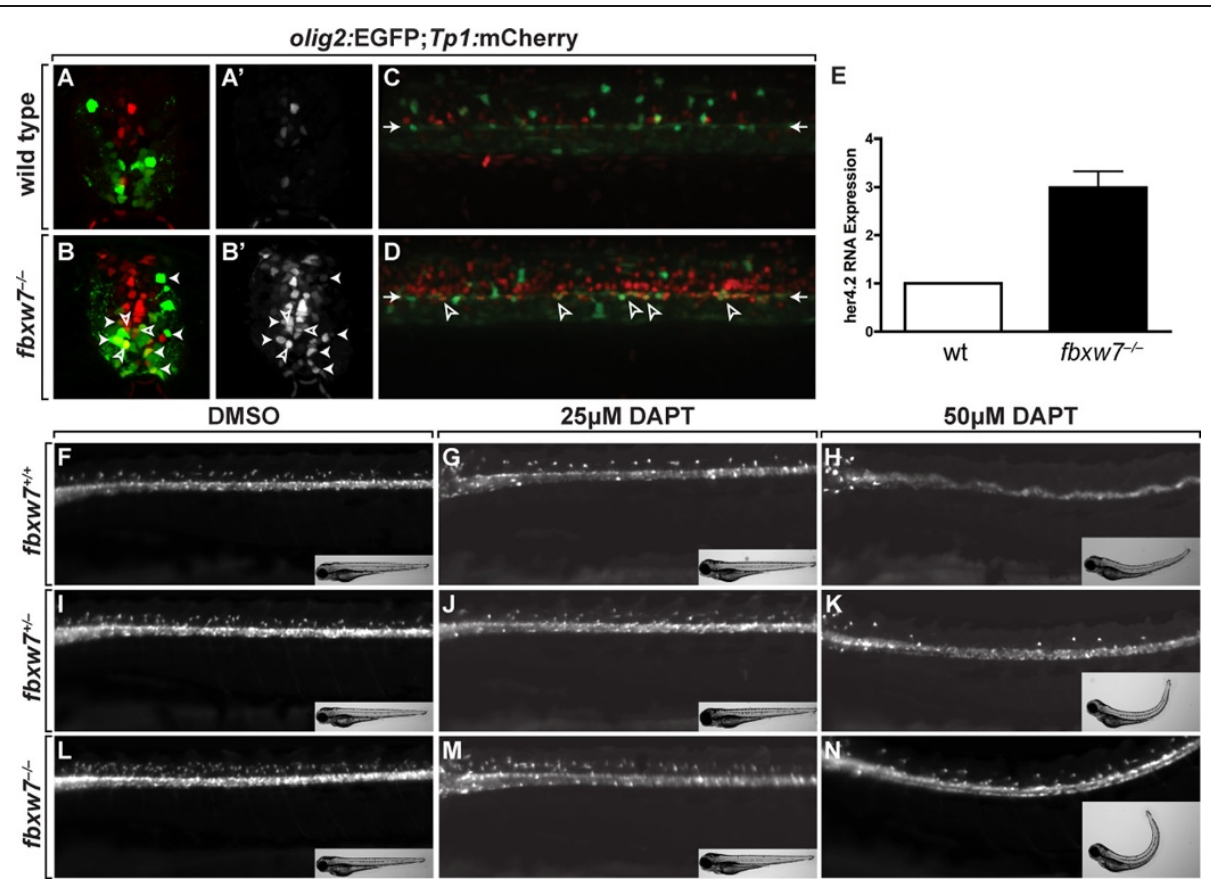

Figure 5 Fbxw7 regulates oligodendrocyte number by regulating Notch signaling. (A,B) Transverse sections of 3 dpf wild-type (A) and fbxw $7^{v 456}$ mutant (B) spinal cords showing Tp 1:mCherry and olig2:EGFP expression. Outlined arrowheads mark Tp1:mCherry ${ }^{+}$, olig2:EGFP ${ }^{+}$ precursors. Solid arrowheads indicate Tp1:mCherry ${ }^{+}$, olig2:EGFP ${ }^{+}$oligodendrocyte lineage cells. $\left(\mathbf{A}^{\prime}, \mathbf{B}^{\prime}\right)$ Tp 1:mCherry images alone. (C,D) Confocal z stack projections, from lateral view, of olig2:EGFP and Tp1:mCherry in $6 \mathrm{dpf}$ wild-type sibling and $f b x w 7^{\text {vu56}}$ mutant larvae. Arrowheads point to row of olig2:EGFP ${ }^{+}$precursors lining the central canal. Outlined arrowheads mark Tp1:mCherry ${ }^{+}$, olig2:EGFP ${ }^{+}$precursors. (E) Relative levels of her4.2 mRNA in $4 \mathrm{dpf}$ wild-type and fbxw $7^{v 156}$ mutant larvae determined by quantitative PCR. Wild-type level was assigned an arbitrary level of 1.0 ( $p=0.0272$ ). (F-N) Lateral images of living $3 \mathrm{dpf}$ larvae treated with DMSO or DAPT plus DMSO from 36-48 hpf. All larvae carried the Tg(olig2:EGFP) reporter to mark OPCs and oligodendrocytes in dorsal spinal cord. DAPT reduced the number of oligodendrocyte lineage cells in larvae of each genotype. Insets show bright field images of larvae. Each larva was genotyped following the experiment.

the spinal cord (Figure $5 \mathrm{C}$ ). olig2:EGFP ${ }^{+}$radial glial precursors, which persist into adulthood [37], were evident as a row of elongated cells at the level of the spinal cord central canal. Few of these cells expressed mCherry, indicating that they had little Notch signaling activity. In fbxw $7^{1-}$ larvae, numerous spinal cord cells expressed mCherry, including olig2: $\mathrm{EGFP}^{+}$radial glial precursors (Figure 5D). We also investigated expression of the Notch target gene her4.2 using quantitative RT-PCR and found that RNA levels were approximately 3-fold higher in $f b x w 7^{/-}$larvae than wild-type larvae at $4 \mathrm{dpf}$ (Figure 5E). Therefore, Notch signaling activity is elevated in the absence of $f b x w 7$ function, consistent with the possibility that Fbxw7 targets Notch for degradation in the zebrafish nervous system.

If elevation of Notch signaling accounts for the excess oligodendrocytes of $f b x w 7^{v u 56}$ mutant larvae, then inhibition of Notch signaling should suppress the mutant phenotype. To test this we used the $\gamma$-secretase inhibitor $N-[N-(3,5-$ difluoro- phenacetyl-1-alanyl)]-S-phenylglycine $t$-butyl ester (DAPT), which prevents release of the signaling-active Notch intracellular domain and produces phenotypes in zebrafish embryos that are indistinguishable from Notch pathway mutations [38]. We applied DAPT to embryos produced by intercrosses of $f b x w 7^{+/-}$adults from 36-48 hpf, the time at which OPCs are specified from neural precursors, assessed the number of dorsally migrated OPCs revealed by $\operatorname{Tg}$ (olig2:EGFP) reporter expression at $72 \mathrm{hpf}$ and genotyped each animal. When exposed to $25 \mu \mathrm{M}$ DAPT, larvae of all three possible genotypes $\left(f b x w 7^{+/+}\right.$, $f b x w 7^{+/-}, f b x w 7^{-/-}$) had normal morphologies and slightly fewer dorsal OPCs than the corresponding genotypes treated only with DMSO (Figure 5G, J, M). 50 $\mu \mathrm{M}$ DAPT frequently produced pronounced morphological defects, including upwardly curled tails, and blocked formation of most OPCs in $f b x w 7^{+/+}$and $f b x w 7^{+/-}$ larvae (Figure $5 \mathrm{H}, \mathrm{K}$ ), consistent with our previous demonstration that Notch signaling is necessary for OPC specification [15]. We found two classes of phenotype among 50 $\mu \mathrm{M}$ DAPT-treated $f b x w 7^{/-}$larvae. $25 \%$ of the homozygous mutant larvae $(n=12)$ had morphological defects and fewer OPCs than control treated $f b x w 7^{+/+}$larvae (Figure $5 \mathrm{~N}$ ). The remaining homozygous mutant larvae had apparently normal morphologies and approximately the same number 
OPCs as control treated $f b x w 7^{+/+}$larvae (data not shown). Therefore, inhibition of Notch signaling suppresses the excess OPC phenotype of $f b x w 7^{v u 56}$ mutant larvae, supporting our hypothesis that Fbxw7 limits the number of OPCs specified from neural precursors by regulating the amount of actively signaling Notch proteins.

\section{Discussion}

During development, dividing neural precursors of the ventral spinal cord give rise first to motor neurons and then oligodendrocytes. The orderly formation of motor neurons and oligodendrocytes requires mechanisms that both maintain dividing neural precursors through neurogenesis and switch them from producing neurons to oligodendrocytes. Notch signaling is essential for balancing neural precursor maintenance with motor neuron and oligodendrocyte specification because mutations that disrupt Notch signaling cause loss of neural precursors, formation of excess early born neurons and a deficit of oligodendrocytes [15]. How Notch signaling is controlled to maintain a precise balance of precursors, neurons and oligodendrocytes is not known. Our work now indicates that negative regulation of Notch activity by Fbxw7 ubiquitin ligase limits the number of neural precursors specified for oligodendrocyte fate.

The founding member of metazoan Fbxw7 genes is nematode sel-10, which was identified as a negative regulator of the Notch homolog lin-12 [39]. Work since then has shown that sel-10/Fbxw7 is the substrate recognition subunit of a SCF ubiquitin ligase that targets Notch proteins for degradation [33-35], as well as several other proteins that are important for cell cycle control [40]. Accordingly, mutation of human $F B X W 7$ has been implicated in numerous cancers $[40,41]$. Substrate recognition by Fbxw7 is dependent upon phosphorylation of specific amino acid sequences called phosphodegrons [40]. The Notch phosphodegron lies within the NICD [42], which is responsible for Notch signal transduction, thereby providing a mechanism for attenuation of Notch signaling activity.

In a previous study we showed that forced expression of NICD in transgenic zebrafish blocked formation of most neurons and produced an excess of OPCs [15] nearly identical to that of $f b x w 7$ mutants. These data implied that Notch signaling must be restricted to properly balance neuron and OPC formation but the mechanisms that attenuate Notch signaling in the nervous system were not clear. The work reported here showed that Notch activity was elevated in neural precursors of $f b x w 7$ mutants and that inhibition of Notch signaling suppressed formation of excess OPCs, indicating that Fbxw7 limits Notch signaling within spinal cord precursors to limit OPC production. These data therefore contribute to growing evidence that Notch activity can be regulated at numerous levels, including at the level of signal attenuation via ubiquitin ligase-mediated degradation [43-46].

Our in vivo time-lapse imaging showed directly that pMN precursors produced excess OPCs in $f b x w 7$ mutant zebrafish embryos. Our data therefore strongly indicate that Fbxw7 controls OPC number by regulating Notch signaling activity in neural precursors. We have previously shown that individual pMN precursors can produce both motor neurons and OPCs in zebrafish [7] and that a deficit of neurons accompanied the formation of excess OPCs resulting from transgene-driven expression of NICD [15]. These data were consistent with a model in which Notch functions as a binary switch, with high signaling levels blocking neurogenesis and promoting oligodendrogenesis. However, we found no decrease in the number of motor neurons in $f b x w 7$ mutant embryos, despite elevated levels of Notch signaling. This raises the possibility that subtle differences in Notch activity can have distinct effects on the fates of neural precursors, consistent with observations that both fewer and excess copies of Notch pathway genes produce mutant phenotypes in flies and vertebrates [47]. Our in situ RNA hybridization experiments revealed no evidence of temporally and spatially regulated $f b x w 7$ expression that could account for a fine-scale modulation of Notch activity in neural precursors. Therefore, the phosphorylation status of Notch phosphodegrons might be a key feature in regulation of $\mathrm{pMN}$ precursors for oligodendrocyte fate.

Two recent investigations of mice in which transgenic expression of Nestin-Cre was used to eliminate Fbxw7 from neural precursors also produced evidence that Notch is an important target of Fbxw7, but additionally arrived at some conclusions that were different from each other and from our own. In one report, Hoeck et al. described mutant mice that that had reduced cellularity throughout the brain, which they attributed to a failure of neural stem cell differentiation and neurogenesis due to elevated Notch signaling, and to elevated neuronal progenitor death, resulting from elevated levels of c-Jun, a known Fbxw7 target [20]. This group reported no difference in the number of cells that expressed markers of astrocytes and oligodendrocytes in E18.5 mutant brains or in neurospheres produced from mutant animals. A second report also described evidence of elevated Notch signaling and reduced neurogenesis, but in contrast did not find an increase in cell death or c-Jun protein levels [21]. Furthermore, Matsumoto et al. documented excess numbers of cells that expressed the astrocyte marker GFAP, which was suppressed in culture by inhibition of Notch signaling but, in contrast to our own work, found no change in the 
numbers of oligodendrocyte lineage cells in mutant P0.5 mutant brains or in cell culture.

One possible explanation for the apparently different effects of $F b x w 7$ mutation in mice and zebrafish is that zebrafish might have a second $f b x w 7$-like gene, which could partially compensate for the loss of function caused by the vu56 mutant allele. However, our queries of the zebrafish genome did not reveal evidence of a duplicated gene. A second possibility is that the relatively small number of OPCs and the ability to observe them directly in zebrafish revealed changes in cell number that were obscured in the mouse studies. OPC number is regulated by cell density in vitro [48] and by limiting amounts of growth factor [49,50] and cell-cell contact in vivo [51]. Consequently, regulation of OPC proliferation following their formation in $F b x w 7$ mutant mice or cell culture might have resulted in normal numbers at the time points chosen for analysis. A third possibility is that different types of $F b x w 7$ mutant alleles might produce different phenotypes. Whereas the mouse alleles were designed to eliminate the F-box domain, the zebrafish $v u 56$ allele is a missense mutation within the WD substrate recognition domain. The majority of human FBXW7 alleles implicated in cancer are missense mutations [41] and a missense mutation created in mouse Fbxw7 was functionally distinct from a null allele [52]. However, a splice-blocking antisense morpholino designed to cause truncation of zebrafish Fbxw7 before the F-box produced a phenotype similar to the $v u 56$ missense allele, although this did not produce a null effect because splicing was not completely blocked.

Regulation of OPC and astrocyte number via Fbxw7mediated inhibition of Notch activity is consistent with other studies that implicated a broad role for Notch signaling in gliogenesis. For example, addition of soluble Notch ligand to neural cell cultures enhanced production of astrocytes [53,54] and Notch signaling directly promoted transcription of the radial glia and astrocyte-associated genes BLBP and GFAP [53,55]. In vivo, transient expression of Notch ligands by newly specified neurons appeared to activate Notch signaling in neighboring precursors, driving them toward astrocyte fate via activation of nuclear factor I (NFI) expression and demethylation of astrocyte specific genes [56]. Expression of NICD or the Notch effector Hey2 (also known as Hesr2) drove formation of Müller glia in zebrafish and mouse retinas [57,58] and NICD produced excess radial glia in mouse forebrain [59]. Although the mechanistic details of oligodendrocyte specification are still poorly understood, Notch signaling similarly promoted formation of oligodendrocytes in zebrafish [15], at least in part by regulating cyclin-dependent kinase inhibitor $1 c$ activity [60]. Our work presented here now provides evidence that Fbxw7 limits the number of OPCs formed by neural precursors by attenuating Notch signaling, thereby opening a new avenue to understanding the mechanisms that specify oligodendrocyte development.

\section{Conclusions}

In this paper we show that mutation of $f b x w 7$, which encodes a subunit of a ubiquitin ligase, resulted in production of excess oligodendrocyte progenitor cells from neural precursors in zebrafish embryos. Notch signaling was elevated in $f b x w 7$ mutant embryos and pharmacological inhibition of Notch signaling suppressed formation of excess oligodendrocyte progenitors indicating that Notch proteins are functionally relevant targets of Fbxw7-mediated ubiquitination during oligodendrocyte specification. Our data provide evidence that negative regulation of Notch activity by protein degradation controls production of appropriate numbers of myelinating glial cells from dividing neural precursors in vertebrate embryos.

\section{Methods}

\section{Zebrafish husbandry}

Embryos were produced by pair wise matings, raised at $28.5^{\circ} \mathrm{C}$ in egg water or embryo medium (EM), and staged to hours post-fertilization (hpf) or days postfertilization (dpf) as previously described [61]. Zebrafish strains used include: $\mathrm{AB}, \mathrm{Tg}$ (olig2:EGFP) [62], $\mathrm{Tg}$ (Tp1bglob:hmgb1-mCherry) ${ }^{j h 11}$ [36] and fbxw $7^{\text {vu56. All }}$ experiments were approved and conducted in accordance with the guidelines set forth by the Institutional Animal Care and Use Committee of the University of Colorado Denver, Anschutz Medical Campus.

\section{Mutant screen}

The vu56 allele was identified in a screen for mutations that altered the number and distribution of OPCs, revealed by $\operatorname{Tg}$ (olig2:EGFP) reporter expression. $\mathrm{AB}$ males were mutagenized with $N$-ethyl $N$-nitrosourea (ENU) as described previously [63]. Mutagenized males were crossed to $\operatorname{Tg}$ (olig2:EGFP) females to create an F1 generation. F1 fish were raised to adulthood and crossed to wild-type $\operatorname{Tg}$ (olig2:egfp) fish to create F2 families. F2 siblings were randomly intercrossed and their progeny screened using fluorescent stereomicroscopes. Identified F2 vu56 heterozygotes were outcrossed to $A B$ fish to propagate the line and to the WIK laboratory strain to create families for genetic mapping. The vu56 allele has been maintained by repeated outcrossing to $A B$ fish.

\section{Mapping and PCR genotyping}

$f b x w 7^{v u 56}$ mutants were identified at $3 \mathrm{dpf}$ and collected with wild-type siblings for isolation of DNA in lysis buffer (10 mM Tris pH 8.0, $50 \mathrm{mM} \mathrm{KCl,} \mathrm{0.3 \%} \mathrm{Tween-20,} \mathrm{0.3 \%}$ $\mathrm{NP}-40$ ) with $1 \mu \mathrm{g} / \mu \mathrm{L}$ Proteinase $\mathrm{K}$ at $55^{\circ} \mathrm{C}$ overnight. 
Pooled DNA was used for bulked-segregant analysis [64] with published simple sequence length polymorphisms (SSLPs) (www.zfin.org). Individual embryos were used to determine recombination frequencies for finer mapping of $f b x w 7^{v u 56}$. The following primers were designed to amplify sequences flanking the mutation for restriction fragment length polymorphism genotyping: $f b x w 7$ forward primer: 5'-CAG TTG ATT TAC CTT TGC GT-3'; reverse primer: 5' -TGT GTC AAT GTG TTT CGG TT-3'. Products were digested with BamHI and HinfI and analyzed using agarose gel electrophoresis.

\section{Isoform cloning and RT-PCR analysis of expression}

We obtained clones corresponding to $f b x w 7 \alpha, \beta$ and $\gamma$ transcripts using PCR to amplify genomic sequences, which were cloned into pCR2.1-TOPO vectors. The following primer pairs were used for amplification: $\alpha$, 759 bp product, 5' -CAGAATGCCAAGTCCTTGTC-3' / 5'-CCTATTCGGTGAGCGAAGG-3'; $\beta, 254$ bp product, 5'-GGCTCAGTCAGTCCGCTCAG-3'/5' -TTTATAGAA GATCATCTTTAAAGTG-3'; $\gamma, 520$ bp product, 5' GCTTGGTGTGAACACTTAAAAC-3' $/ 5^{\prime}$-CATAATTG CATCATTTCCACATT-3'. To investigate expression at different developmental stages we used the isoform specific forward primers with the reverse primer 5'-CGT CGT CTC TGT GGA ACC-3' from the common region to amplify cDNA from single-cell, $24 \mathrm{hpf}$, and $3 \mathrm{dpf}$ embryos.

\section{Immunohistochemistry}

Embryos were fixed using 4\% paraformaldehyde, embedded, frozen and sectioned using a cryostat microtome as previously described [15]. We used the following primary antibodies: rabbit anti-Sox10 (1:500) [60], mouse anti-Isl (39.4D5, Developmental Studies Hybridoma Bank (DSHB) Iowa City, Iowa USA, 1:100), mouse anti-BrdU (G3G40, DSHB, 1:10), and anti-Sox2 (ab97959, Abcam, 1:1000). For fluorescent detection of antibody labeling, we used Alexa Fluor 568 goat anti-mouse or goat anti-rabbit conjugates (Invitrogen, 1:200). Images were captured using either a Zeiss Axiovert 200 inverted microscope equipped with a PerkinElmer Ultraview ERS Live Cell Imager spinning disc confocal system or a Zeiss AxioObserver inverted microscope equipped with a PerkinElmer UltraVIEW VoX confocal system and analyzed with Volocity software (PerkinElmer) and Adobe Photoshop. Image adjustments were limited to contrast enhancement, level settings, auto tone and cropping.

\section{In situ RNA hybridization}

The following RNA probes were generated for this manuscript: $f b x w 7$, which recognizes all isoforms, from an EST (EB835996), $\alpha-f b x w 7, \beta-f b x w 7$, and $\gamma$-fbxw7 isoform specific probes from 24 hpf cDNA, and plpla and $m b p$ [65]. in situ RNA hybridization was performed according to published methods [66]. Embryos were either mounted for whole-mount imaging or embedded and sectioned as above. Images were captured using either an Olympus AX70 microscope equipped with DIC optics, a Retiga Exicooled CCD camera (QImaging) and Openlab software (Improvision) or a similarly equipped Zeiss AxioObserver inverted microscope and Volocity software (Improvision). Image data were exported to Adobe Photoshop and adjustments were limited to level settings, color balance and cropping.

\section{Quantitative PCR}

RNA was isolated from 3 sets of 20 pooled wild-type larvae and 3 sets of 20 pooled $f b x w 7^{-1-}$ larvae at $4 \mathrm{dpf}$. Reverse Transcriptase was performed using Superscript III First Strand Synthesis for qPCR (Invitrogen). Real-time qPCR was performed on each sample in triplicate using an Applied Biosystems StepOne Plus machine and software version 2.1. Taqman Gene Expression Assays (Applied Biosystems) were used to detect her4.2 (Dr03160688_g1) and bactin1 (Dr03432610_m1).

\section{In vivo time-lapse imaging}

Embryos were lightly anesthetized using Tricaine, mounted on their sides in $0.7 \%$ low-melting temperature agarose in $35 \mathrm{~mm}$ glass bottom dishes and covered with EM containing Tricaine. Z-stack images were captured every 15 minutes for 24 hours using an inverted Zeiss AxioObserver equipped with motorized and heated stage and a PerkinElmer UltraVIEW VoX confocal system. The imaging chamber was maintained at $28.5^{\circ} \mathrm{C}$. $4 \mathrm{D}$ data sets were analyzed using Volocity software (PerkinElmer) and movies were exported to QuickTime. Image adjustments were limited to contrast enhancements and cropping frame size.

\section{Morpholino injections}

$f b x w 7^{S S M O}$, consisting of the sequence $5^{\prime}$-GCCAACTACAACAAGACAGAGACAG-3' (Gene Tools, LLC) was designed to have sequence complementary to the boundary of intron 4 and exon 5 of $f b x w 7$. The MO was resuspended in sterile water to a stock concentration of $1 \mathrm{mM}$ and stored at room temperature. The stock was diluted in 2X injection buffer $(240 \mathrm{mM} \mathrm{KCl}, 40 \mathrm{mM}$ HEPES, and $0.5 \%$ Phenol red) to a concentration of $0.125 \mathrm{mM}$ and $2 \mathrm{~nL}$ was injected into the yolk of one- to two-cell stage embryos.

\section{DAPT treatments}

The $\gamma$-secretase inhibitor $N$-[N-(3,5-difluoro- phenacetyll-alanyl)]-S-phenylglycine $t$-butyl ester (DAPT) (Calbiochem) was resuspended in dimethyl sulfoxide (DMSO) to a stock concentration of $20 \mathrm{mM}$ and stored in aliquots at $-20^{\circ} \mathrm{C}$. Embryos were manually dechorionated at 36 
hpf and placed in $25 \mu \mathrm{M}$ or $50 \mu \mathrm{M}$ in EM with $1 \%$ DMSO for 12 hours at $28.5^{\circ} \mathrm{C}$. DAPT solution was replaced with EM and embryos were allowed to develop to $3 \mathrm{dpf}$ at $28.5^{\circ} \mathrm{C}$. At $3 \mathrm{dpf}$ embryos were imaged individually using a stereomicroscope equipped with bright field and epifluorescence optics followed by DNA isolation and $f b x w 7^{v u 56}$ genotyping as described above.

\section{BrdU labeling}

Dechorionated embryos were labeled with 5-bromo-2'deoxyuadine (BrdU) (Roche) by incubating them in $20 \mathrm{mM}$ BrdU in EM with 10\% DMSO at room temperature for $30 \mathrm{~min}$. The embryos were then rinsed and maintain at room temperature for $30 \mathrm{~min}$ and then fixed using 4\% paraformaldehyde. After embedding and sectioning as described above, the tissue sections were treated with $2 \mathrm{~N} \mathrm{HCl}$ for $30 \mathrm{~min}$ before processing for anti-BrdU immunohistochemistry.

\section{Data quantification and statistical analysis}

Cell counts were obtained by direct observation of sections using the microscopes described above. For Sox10, $m b p$, and plp1a quantification, 10 sections per embryo were counted to produce the average number per section. For Isl quantification, 9 sections per embryo were counted to produce the average number per section. GraphPad Prism software was used for statistical analysis.

\section{Abbreviations \\ OPCs: Oligodendrocyte progenitor cells; CNS: Central nervous system; NICD: Notch intracellular domain; dpf: Days post fertilization; hpf: Hours post fertilization; SSLPs: Simple sequence length polymorphisms; PCR: Polymerase chain reaction; RFLP: Restriction fragment length polymorphism; MO: Morpholino oligonucleotide; ng: Nanogram; EST: Expressed sequence tag; PCR: Polymerase chain reaction; RACE: Rapid amplification of CDNA ends: DAPT, $N$-[N-(3,5-difluoro- phenacetyl-l-alanyl)]-S-phenylglycine $t$-butyl ester; DMSO: dimethyl sulfoxide; bp: Base pairs.}

\section{Competing interests}

The authors declare that they have no competing interests.

\begin{abstract}
Acknowledgments
This work was supported by National Institutes of Health Grant NS046668, Training Program in Developmental Biology T32 HD007502 (J.L.S.) and Gates Frontiers Fund. Zebrafish housing and care was supported by the Rocky Mountain Neurological Disorders Core Center (P30 NS048154). The anti-BrdU antibody, developed by S. J. Kaufman, and the anti-Isl antibody, developed by T. M. Jessell, were obtained from the Developmental Studies Hybridoma Bank developed under the auspices of the National Institute of Child Health and Human Development and maintained by The University of lowa, Department of Biological Sciences, lowa City, IA. We thank Danette Nicolay for help with quantitative PCR, Macie Walker for cloning isoform-specific DNA and members of the Appel lab and Wendy Macklin for discussions and Jacob Hines for comments on the manuscript.
\end{abstract}

\section{Author details}

${ }^{1}$ Department of Biological Sciences, Vanderbilt University, Nashville, TN, USA ${ }^{2}$ Departments of Pediatrics and Cell and Developmental Biology, University of Colorado School of Medicine, Aurora, CO, USA. ${ }^{3}$ University of Colorado School of Medicine, MS 8108, Aurora, CO 80045, USA.

\section{Authors' contributions}

$J L S$ performed all the experiments described in this manuscript except for quantitative PCR and Sox2 and BrdU immunohistochemistry, which was performed by CAK. BA conceived of the study and helped design and interpret experiments. JLS and BA wrote the manuscript. All authors read and approved the manuscript.

Received: 3 January 2012 Accepted: 3 May 2012

Published: 3 May 2012

\section{References}

1. Takebayashi H, Yoshida S, Sugimori M, Kosako H, Kominami R, Nakafuku M, Nabeshima Y: Dynamic expression of basic helix-loop-helix Olig family members: implication of Olig2 in neuron and oligodendrocyte differentiation and identification of a new member, Olig3. Mech Dev 2000, 99:143-148.

2. Lu QR, Yuk D, Alberta JA, Zhu Z, Pawlitzky I, Chan J, Mcmahon AP, Stiles CD, Rowitch DH: Sonic hedgehog-regulated oligodendrocyte lineage genes encoding bHLH proteins in the mammalian central nervous system. Neuron 2000, 25:317-329.

3. Zhou Q, Wang S, Anderson DJ: Identification of a novel family of oligodendrocyte lineage-specific basic helix-loop-helix transcription factors. Neuron 2000, 25:331-343.

4. Lu QR, Sun T, Zhu Z, Ma N, Garcia M, Stiles CD, Rowitch DH: Common developmental requirement for Olig function indicates a motor neuron/ oligodendrocyte connection. Cell 2002, 109:75-86.

5. Zhou Q, Anderson DJ: The bHLH transcription factors OLIG2 and OLIG1 couple neuronal and glial subtype specification. Cell 2002, 109:61-73.

6. Masahira N, Takebayashi H, Ono K, Watanabe K, Ding L, Furusho M, Ogawa Y, Nabeshima Y, Alvarezbuylla A, Shimizu K: Olig2-positive progenitors in the embryonic spinal cord give rise not only to motoneurons and oligodendrocytes, but also to a subset of astrocytes and ependymal cells. Dev Biol 2006, 293:358-369.

7. Park H-C, Shin J, Appel B: Spatial and temporal regulation of ventral spinal cord precursor specification by Hedgehog signaling. Development 2004, 131:5959-5969.

8. Zhou Q, Choi G, Anderson DJ: The bHLH transcription factor Olig2 promotes oligodendrocyte differentiation in collaboration with Nkx2.2. Neuron 2001, 31:791-807

9. Stolt CC: The Sox9 transcription factor determines glial fate choice in the developing spinal cord. Genes Dev 2003, 17:1677-1689.

10. Deneen B, Ho R, Lukaszewicz A, Hochstim CJ, Gronostajski RM, Anderson DJ: The transcription factor NFIA controls the onset of gliogenesis in the developing spinal cord. Neuron 2006, 52:953-968.

11. Imayoshi I, Sakamoto M, Yamaguchi M, Mori K, Kageyama R: Essential roles of Notch signaling in maintenance of neural stem cells in developing and adult brains. J Neurosci 2010, 30:3489-3498.

12. La Pompa De JL, Wakeham A, Correia KM, Samper E, Brown S, Aguilera RJ, Nakano T, Honjo T, Mak TW, Rossant J, Conlon RA: Conservation of the Notch signalling pathway in mammalian neurogenesis. Development 1997, 124:1139-1148.

13. Chitnis A, Henrique D, Lewis J, Ish-Horowicz D, Kintner C: Primary neurogenesis in Xenopus embryos regulated by a homologue of the Drosophila neurogenic gene Delta. Nature 1995, 375:761-766.

14. Appel B, Eisen JS: Regulation of neuronal specification in the zebrafish spinal cord by Delta function. Development 1998, 125:371-380.

15. Park H-C, Appel B: Delta-Notch signaling regulates oligodendrocyte specification. Development 2003, 130:3747-3755

16. Itoh M, Kim C-H, Palardy G, Oda T, Jiang Y-J, Maust D, Yeo S-Y, Lorick K Wright GJ, Ariza-McNaughton L, Weissman AM, Lewis J, Chandrasekharappa SC, Chitnis AB: Mind bomb is a ubiquitin ligase that is essential for efficient activation of Notch signaling by Delta. Dev Cell 2003, 4:67-82.

17. Appel B, Givan LA, Eisen JS: Delta-Notch signaling and lateral inhibition in zebrafish spinal cord development. BMC Dev Biol 2001, 1:13.

18. Yang $X$, Tomita T, Wines-Samuelson M, Beglopoulos V, Tansey MUG, Kopan $R$, Shen J: Notch1 signaling Influences V2 interneuron and motor neuron development in the spinal cord. Dev Neurosci 2006, 28:102-117.

19. Jandke A, Da Costa C, Sancho R, Nye E, Spencer-Dene B, Behrens A: The $\mathrm{F}$-box protein Fbw7 is required for cerebellar development. Dev Biol 2011, 358:201-212. 
20. Hoeck JD, Jandke A, Blake SM, Nye E, Spencer-Dene B, Brandner S, Behrens $A$ : Fbw7 controls neural stem cell differentiation and progenitor apoptosis via Notch and c-Jun. Nat Neurosci 2010, 13:1365-13651372.

21. Matsumoto A, Onoyama I, Sunabori T, Kageyama R, Okano H, Nakayama Kl: Fbxw7-dependent degradation of Notch is required for control of "stemness" and neuronal-glial differentiation in neural stem cells. J Biol Chem 2011, 286:13754-13764.

22. Park H: olig2 is required for Zebrafish primary motor neuron and oligodendrocyte development. Dev Biol 2002, 248:356-368.

23. Hagedorn M, Delugin M, Abraldes I, Allain N, Belaud-Rotureau M-A, Turmo M, Prigent C, Loiseau H, Bikfalvi A, Javerzat S: FBXW7/hCDC4 controls glioma cell proliferation in vitro and is a prognostic marker for survival in glioblastoma patients. Cell Div 2007, 2:9.

24. Gu Z, Inomata K, Ishizawa K, Horii A: The FBXW7 beta-form is suppressed in human glioma cells. Biochem Biophys Res Commun 2007, 354:992-998.

25. Ng PC, Henikoff S: Predicting deleterious amino acid substitutions. Genome Res 2001, 11:863-874.

26. Ramensky V, Bork P, Sunyaev S: Human non-synonymous SNPs: server and survey. Nucleic Acids Res 2002, 30:3894-3900.

27. Spruck CH, Strohmaier H, Sangfelt O, Müller HM, Hubalek M, Müller-Holzner E, Marth C, Widschwendter M, Reed SI: hCDC4 gene mutations in endometrial cancer. Cancer Res 2002, 62:4535-4539.

28. Matsumoto A, Onoyama I, Nakayama Kl: Expression of mouse Fbxw7 isoforms is regulated in a cell cycle- or p53-dependent manner. Biochem Biophys Res Commun 2006, 350:114-119.

29. Welcker M: A Nucleolar Isoform of the Fbw7 Ubiquitin Ligase Regulates c-Myc and Cell Size. Curr Biol 2004, 14:1852-1857.

30. Almeida AD, Wise HM, Hindley CJ, Slevin MK, Hartley RS, Philpott A: The F-box protein Cdc4/Fbxw7 is a novel regulator of neural crest development in Xenopus laevis. Neural Dev 2010, 5:1.

31. Takada N, Kucenas S, Appel B: Sox10 is necessary for oligodendrocyte survival following axon wrapping. Glia 2010, 58:996-1006.

32. Pevny LH, Nicolis SK: Sox2 roles in neural stem cells. Int J Biochem Cell Biol 2010, 42:421-424.

33. Oberg C, Li J, Pauley A, Wolf E, Gurney M, Lendahl U: The Notch intracellular domain is ubiquitinated and negatively regulated by the mammalian Sel-10 homolog. J Biol Chem 2001, 276:35847-35853.

34. Wu G, Lyapina S, Das I, Li J, Gurney M, Pauley A, Chui I, Deshaies RJ, Kitajewski J: SEL-10 is an inhibitor of notch signaling that targets notch for ubiquitin-mediated protein degradation. Mol Cell Biol 2001, 21:7403-7415

35. Gupta-Rossi N, Le Bail O, Gonen H, Brou C, Logeat F, Six E, Ciechanover A, Israël A: Functional interaction between SEL-10, an F-box protein, and the nuclear form of activated Notch1 receptor. J Biol Chem 2001, 276:34371-34378

36. Parsons MJ, Pisharath $H$, Yusuff S, Moore JC, Siekmann AF, Lawson N, Leach SD: Notch-responsive cells initiate the secondary transition in larval zebrafish pancreas. Mech Dev 2009, 126:898-912.

37. Park H-C, Shin J, Roberts RK, Appel B: Anolig2 reporter gene marks oligodendrocyte precursors in the postembryonic spinal cord of zebrafish. Dev Dyn 2007, 236:3402-3407.

38. Geling A, Steiner H, Willem M, Bally-Cuif L, Haass C: A gamma-secretase inhibitor blocks Notch signaling in vivo and causes a severe neurogenic phenotype in zebrafish. EMBO Rep 2002, 3:688-694.

39. Hubbard EJ, Wu G, Kitajewski J, Greenwald I: sel-10, a negative regulator of lin-12 activity in Caenorhabditis elegans, encodes a member of the CDC4 family of proteins. Genes Dev 1997, 11:3182-3193.

40. Welcker M, Clurman BE: FBW7 ubiquitin ligase: a tumour suppressor at the crossroads of cell division, growth and differentiation. Nat Rev Cancer $2008,8: 83-93$.

41. Akhoondi S, Sun D, von der Lehr N, Apostolidou S, Klotz K, Maljukova A, Cepeda D, Fiegl H, Dafou D, Dofou D, Marth C, Mueller-Holzner E, Corcoran M, Dagnell M, Nejad SZ, Nayer BN, Zali MR, Hansson J, Egyhazi S, Petersson F, Sangfelt P, Nordgren H, Grander D, Reed SI, Widschwendter M, Sangfelt O, Spruck C: FBXW7/hCDC4 is a general tumor suppressor in human cancer. Cancer Res 2007, 67:9006-9012.

42. O'Neil J, Grim J, Strack P, Rao S, Tibbitts D, Winter C, Hardwick J, Welcker M, Meijerink JP, Pieters R, Draetta G, Sears R, Clurman BE, Look AT: FBW7 mutations in leukemic cells mediate NOTCH pathway activation and resistance to -secretase inhibitors. J Exp Med 2007, 204:1813-1824.
43. Lai EC: Protein degradation: four E3s for the notch pathway. Curr Biol 2002, 12:R74-R78.

44. Fior R, Fior R, Henrique D, Henrique D: "Notch-Off": a perspective on the termination of Notch signalling. Int J Dev Biol 2009, 53:1379-1384.

45. Fortini ME: Notch signaling: the core pathway and its posttranslational regulation. Dev Cell 2009, 16:633-647.

46. Kopan R, llagan MXG: The canonical Notch signaling pathway: unfolding the activation mechanism. Cell 2009, 137:216-233.

47. Andersson ER, Sandberg R, Lendahl U: Notch signaling: simplicity in design, versatility in function. Development 2011, 138:3593-3612.

48. Zhang $\mathrm{H}$, Miller $\mathrm{RH}$ : Density-dependent feedback inhibition of oligodendrocyte precursor expansion. J Neurosci 1996, 16:6886-6895.

49. van Heyningen P, Calver AR, Richardson WD: Control of progenitor cell number by mitogen supply and demand. Curr Biol 2001, 11:232-241.

50. Calver AR, Hall AC, Yu WP, Walsh FS, Heath JK, Betsholtz C, Richardson WD: Oligodendrocyte population dynamics and the role of PDGF in vivo. Neuron 1998, 20:869-882.

51. Kirby BB, Takada N, Latimer AJ, Shin J, Carney TJ, Kelsh RN, Appel B: In vivo time-lapse imaging shows dynamic oligodendrocyte progenitor behavior during zebrafish development. Nat Neurosci 2006, 9:1506-1511.

52. Davis H, Lewis A, Spencer-Dene B, Tateossian H, Stamp G, Behrens A, Tomlinson I: FBXW7 mutations typically found in human cancers are distinct from null alleles and disrupt lung development. J Pathol 2011, 224:180-189.

53. Ge W, Martinowich K, Wu X, He F, Miyamoto A, Fan G, Weinmaster G, Sun YE: Notch signaling promotes astrogliogenesis via direct CSL-mediated glial gene activation. J Neurosci Res 2002, 69:848-860.

54. Grandbarbe L: Delta-Notch signaling controls the generation of neurons/ glia from neural stem cells in a stepwise process. Development 2003 130:1391-1402.

55. Anthony TE: Brain lipid-binding protein is a direct target of Notch signaling in radial glial cells. Genes Dev 2005, 19:1028-1033.

56. Namihira M, Kohyama J, Semi K, Sanosaka T, Deneen B, Taga T, Nakashima K Committed neuronal precursors confer astrocytic potential on residual neural precursor cells. Dev Cell 2009, 16:245-255.

57. Scheer N, Groth A, Hans S, Campos-Ortega JA: An instructive function for Notch in promoting gliogenesis in the zebrafish retina. Development 2001 128:1099-1107.

58. Satow T, Bae SK, Inoue T, Inoue C, Miyoshi G, Tomita K, Bessho Y, Hashimoto $\mathrm{N}$, Kageyama R: The basic helix-loop-helix gene hesr2 promotes gliogenesis in mouse retina. J Neurosci 2001, 21:1265-1273.

59. Gaiano N, Nye J, Fishell G: Radial Glial Identity Is Promoted by Notch1 Signaling in the Murine Forebrain. Neuron 2000, 25:395-395404.

60. Park H-C, Boyce J, Shin J, Appel B: Oligodendrocyte specification in zebrafish requires notch-regulated cyclin-dependent kinase inhibitor function. J Neurosci 2005, 25:6836-6844.

61. Kimmel CB, Ballard WW, Kimmel SR, Ullmann B, Schilling TF: Stages of embryonic development of the zebrafish. Dev Dyn 1995, 203:253-310.

62. Shin J, Park H-C, Topczewska JM, Mawdsley DJ, Appel B: Neural cell fate analysis in zebrafish using olig2 BAC transgenics. Methods Cell Sci 2003, 25:7-14

63. Solnica-Krezel $L$, Schier AF, Driever W: Efficient recovery of ENU-induced mutations from the zebrafish germline. Genetics 1994, 136:1401-1420.

64. Postlethwait $J \mathrm{H}$, Talbot WS: Zebrafish genomics: from mutants to genes. Trends Genet 1997, 13:183-190.

65. Brosamle C, Halpern M: Characterization of myelination in the developing zebrafish. Glia 2002, 39:45-57.

66. Hauptmann G, Gerster T: Multicolor whole-mount in situ hybridization. Methods Mol Biol 2000, 137:139-148.

doi:10.1186/1749-8104-7-15

Cite this article as: Snyder et al.: Fbxw7 regulates Notch to control specification of neural precursors for oligodendrocyte fate. Neural Development 2012 7:15. 